\title{
Optical observations of cataclysmic variables discovered by INTEGRAL
}

\section{Magaretha L. Pretorius*}

South African Astronomical Observatory, PO Box 9, Observatory 7935, Cape Town

E-mail: retha@saao.ac.za

\begin{abstract}
A large fraction of the cataclysmic variable stars (CVs) detected by INTEGRAL consists of intrinsically rare systems, specifically asynchronous polars and intermediate polars (IPs). Surveys at lower energies have produced relatively fewer objects in these two classes. It is clear that INTEGRAL is detecting preferentially rare and interesting CVs. However, while it has been speculated that many of the CVs discovered by INTEGRAL are IPs, very little is known about most of these systems. In order to address this, I present time-resolved optical observations of several CVs discovered by INTEGRAL. While the white dwarf spin modulation is detected in high-speed photometry of three of the new CVs, two others show no evidence of magnetism, and should be considered non-magnetic systems.
\end{abstract}

7th INTEGRAL Workshop

September 8-11 2008

Copenhagen, Denmark

\footnotetext{
* Speaker.
} 


\section{Introduction}

Cataclysmic variables (CVs) are semi-detached interacting binaries, in which a white dwarf primary star accretes from a low-mass, approximately main-sequence companion (see [1] for the most comprehensive review of the subject). The mass transfer is caused by orbital angular momentum loss, and usually leads to the formation of an accretion disc.

In about $20 \%$ of CVs with known orbital periods $\left(P_{o r b}\right)$, the white dwarf has a magnetic field sufficiently strong either to prevent the formation of a disc entirely, or at least disrupt it to a significant extent. These are the magnetic CVs, and they are divided into two sub-types, namely polars and intermediate polars (IPs). Polars show strong circular and linear polarization modulated at $P_{\text {orb }}$ (implying that the white dwarf rotation is synchronized with the binary orbit), while IPs are characterized by very stable pulsations, at periods $<P_{\text {orb }}$ in their optical and X-ray light curves (interpreted as the white dwarf spin modulation). Reviews of polars and IPs may be found in [2] and [3]. The distinction between the observational properties of polars and IPs is explained by a combination of white dwarf magnetic field strength and accretion rate, with polars having stronger fields and lower mass transfer rates $(\dot{M})$ than IPs.

Polars do not have discs - the accretion is magnetically channelled onto small (on the order of 0.01 or 0.001 of the white dwarf surface area; e.g., [4]; [5]) accretion zones near the white dwarf magnetic poles. The optical light is dominated by cyclotron emission (hence the strong polarization), and cyclotron humps are usually detected in high-S/N spectra of polars (e.g. [6]; [7]; [8]).

Accretion exerts a spin-up torque on the rotation of the white dwarf, and it is the strong interaction of the white dwarf and secondary magnetic fields that phase-locks the rotation of the white dwarf in a polar (see e.g. [9]; [10]). In fact, several polars rotate slightly asynchronously (see e.g. [11]; [12]), but the degree of asynchronism in these systems is small (the white dwarf spin period and $P_{\text {orb }}$ differ by only $\sim 1 \%$ ), and they will attain spin-orbit synchronism on a short time-scale (e.g. [13]).

The defining short-period photometric pulsations observed in IPs are explained by magnetically channelled accretion from the inner edge of a truncated disc onto a rapidly rotating white dwarf. The white dwarf spin cycle produces the photometric modulation that characterizes an IP as the rotating 'beam', originating from the anisotropic accretion flow, periodically sweeps either across the line of sight, or over some structure that reprocesses it (see e.g. [14]; [15]; [16]; [17]).

The most direct evidence supporting the magnetic rotator model of IPs is the detection of circular polarization in several systems ([18]; [19]; [20]; [21]).

IPs have harder X-ray spectra than polars, but both polars and IPs have large X-ray to optical flux ratios compared to non-magnetic CVs. High excitation lines, specifically $\mathrm{He}$ II $\lambda 4686$, are also prominent in the optical spectra of magnetic CVs.

The INTEGRAL IBIS/ISGRI Soft Gamma-Ray Survey has for the first time systematically imaged a large fraction of the entire sky at energies above $20 \mathrm{keV}$ (e.g. [22]; [23]). Roughly 5\% of sources detected in this survey are CVs, and a large fraction these consists of intrinsically rare systems. Specifically, 2 INTEGRAL CVs are asynchronous polars (there are only 6 known), and roughly half the CVs detected by INTEGRAL are IPs. Overviews of the CV sample constructed from observations in this band may be found in [24] and [25]. 
INTEGRAL is certainly detecting preferentially rare and interesting CVs. However, while several of the systems discovered by INTEGRAL have been demonstrated to be IPs (e.g., [26]; [27]; [28]; [29]), very little is known about others. In order to address this, follow-up observations in the optical or softer X-ray bands are needed. This contribution presents time-resolved optical photometry and spectroscopy of five CVs discovered by INTEGRAL.

\section{Target sample}

Five southern sources for which no time-resolved optical data exist were selected for this study; they are listed in Table 1. XSS J12270-4859, IGR J15094-6649, IGR J16167-4957, and IGR J17195-4100 were identified by [30]; the discovery spectrum of IGR J16500-3307 is presented by [31]. [32] shows Swift spectra of XSS J12270-4859, IGR J16167-4957, IGR J16500-3307, and IGR J17195-4100.

Based only on their detection by INTEGRAL and the presence of He II $\lambda 4686$ emission lines in their optical spectra, these $5 \mathrm{CVs}$ were all classified as IPs. However, neither of the above observational properties definitively points to an IP nature. Until the white dwarf spin modulation (or evidence of cyclotron emission) is detected, an IP classification is tentative at best.

The RXTE observations of [33] reveal candidates white dwarf spin modulations in XSS J122704859 and IGR J17195-4100. This is discussed further in Section 4.1.1 and 4.2.3.

Table 1: The targets for this study. Orbital period measurements are listed, but will be described elsewhere. The possible white dwarf spin period listed for XSS J12270-4859 is taken from [33].

\begin{tabular}{llllll}
\hline Object & ROSAT counterpart & Type & $P_{\text {orb }} / \mathrm{h}$ & $P_{1} / \mathrm{min}$ & $V$ \\
\hline XSS J12270-4859 & 1RXS J122758.8-485343 & IP? & - & $14.33 ?$ & 16.1 \\
IGR J15094-6649 & 1RXS J150925.7-664913 & IP & 5.89 & 13.490 & 14.7 \\
IGR J16167-4957 & 1RXS J161637.2-495847 & - & 5.004 & - & 16.5 \\
IGR J16500-3307 & 1RXS J164955.1-330713 & IP & 3.617 & 9.960 & 16.9 \\
IGR J17195-4100 & 1RXS J171935.6-410054 & IP & 4.005 & 18.993 & 15.2 \\
\hline
\end{tabular}

\section{Observations}

Follow-up observations of the five CVs mentioned in Section 2 were obtained from the Sutherland site of the South African Astronomical Observatory (SAAO). The aim of the spectroscopy and photometry was to measure orbital periods and detect white dwarf spin modulations, respectively.

\subsection{High-speed photometry}

I obtained high-speed photometry of all five targets, using the University of Cape Town CCD photometer (UCT CCD; see [34]) on the SAAO 1-m and 0.76-m telescopes. These observations were made in white light, producing photometry with an effective wavelength similar to Johnson $V$, but with a very broad bandpass. This means that the data cannot be precisely placed on a standard photometric system; the magnitude calibration approximates Johnson $V$ to within only $\simeq 0.1$ mag. 
A few examples of the light curves obtained are shown in Fig. 1; every systems was observed on at least 3 nights. The systems all display rapid flickering (the observational signature of mass transfer). Three of the five targets also show coherent short-period modulations.

Approximate average $V$ magnitudes are listed in Table 1 (the targets showed no large systematic variations from night to night), together with the periods interpreted as white dwarf spin periods $\left(P_{1}\right)$ for the three systems that were found to be IPs.

\subsection{Spectroscopy}

In addition to the photometry, I obtained medium-resolution spectra of each object with the SAAO 1.9-m telescope. The Grating Spectrograph equipped with the no. 7 grating and a slit width of 1 !" 8 was used, yielding spectral resolution of $\simeq 5 \AA$ over the wavelength range 3600 to $7200 \AA$.

Each object spectrum was bracketed with arc lamp exposures to provide wavelength calibration. The flux calibration was achieved by observing spectrophotometric standard stars from [35]. Note, however, that these spectra were obtained under non-photometric conditions; the absolute flux calibrations are therefore not reliable. The data were reduced using standard procedures in IRAF $^{1}$, including optimal extraction [36].

The spectra are displayed in Fig. 2; all systems show the broad Balmer, He I, and He II emission lines commonly detected in CVs.

In order to measure orbital periods, I also obtained time-resolved spectroscopy of 4 systems (IGR J15094-6649, IGR J16167-4957, IGR J16500-3307, and IGR J17195-4100) at slightly higher resolution $(\simeq 2 \AA)$. These observations will be described elsewhere; the periods are listed in Table 1 .

\section{Results}

\subsection{Systems that show no evidence of magnetism}

Despite obtaining long light curves of good quality of both XSS J12270-4859 and IGR J161674957 , no coherent modulations were detected in the photometry of these two objects. The spectra of these systems both show He II $\lambda 4686$ emission, but are not helpful in determining the CV subtypes.

\subsubsection{XSS J12270-4859}

High-speed photometry of XSS J12270-4859, covering a total of $11 \mathrm{~h}$, was taken with the 1-m telescope. The data were searched for periodic signals without success. The light curves show nothing other than quite large amplitude (a range of $>1 \mathrm{mag}$ ) flickering.

[33] report a signal at $859.6 \mathrm{~s}$ in several hours of RXTE data of XSS J12270-4859. I detect no significant optical signal with a period near this value. Since quasi-coherent oscillations are common in non-magnetic CVs (see e.g. [37]; [38]; [39]; [40]), the period seen by [33] needs to be observed again before it will be clear whether it has sufficient coherence to be associated with the spin of the white dwarf.

The spectrum of XSS J12270-4859 has broad, but quite weak, emission lines. I did not obtain time-resolved spectroscopy of this system, and the photometry does not reveal the orbital period. XSS J12270-4859 was at similar brightness to what [30] found, during all my observations.

\footnotetext{
${ }^{1}$ IRAF is distributed by the National Optical Astronomy Observatories.
} 


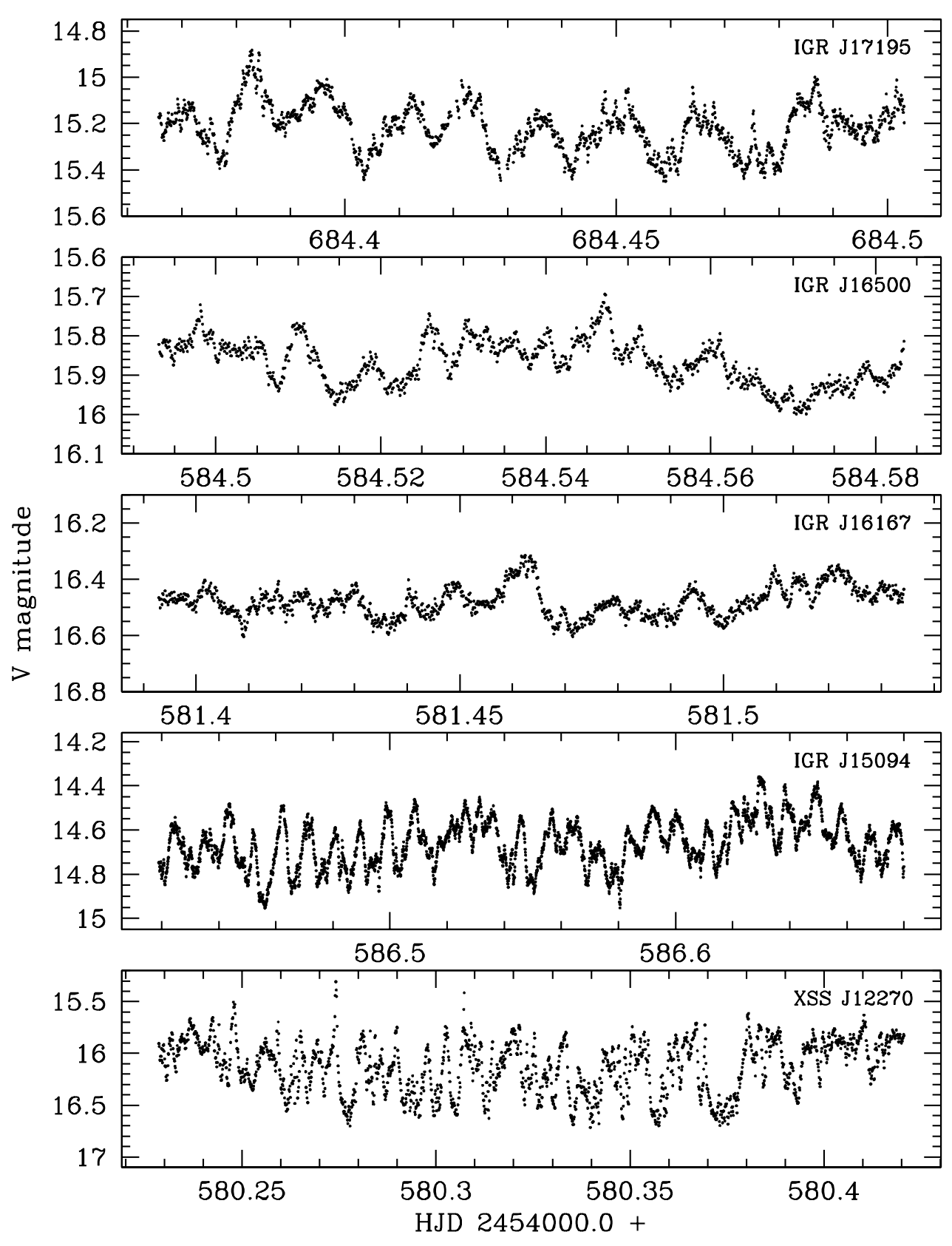

Figure 1: Examples of the light curves of the five objects included in this study. The white dwarf spin pulsations are particularly prominent in the data shown for IGR J15094-6649 and IGR J17195-4100. Typical exposure times where $8 \mathrm{~s}$ (the longest where $10 \mathrm{~s}$ ), each target was observed on 3 or more nights, and the shortest total time spent on an object was $11 \mathrm{~h}$. 


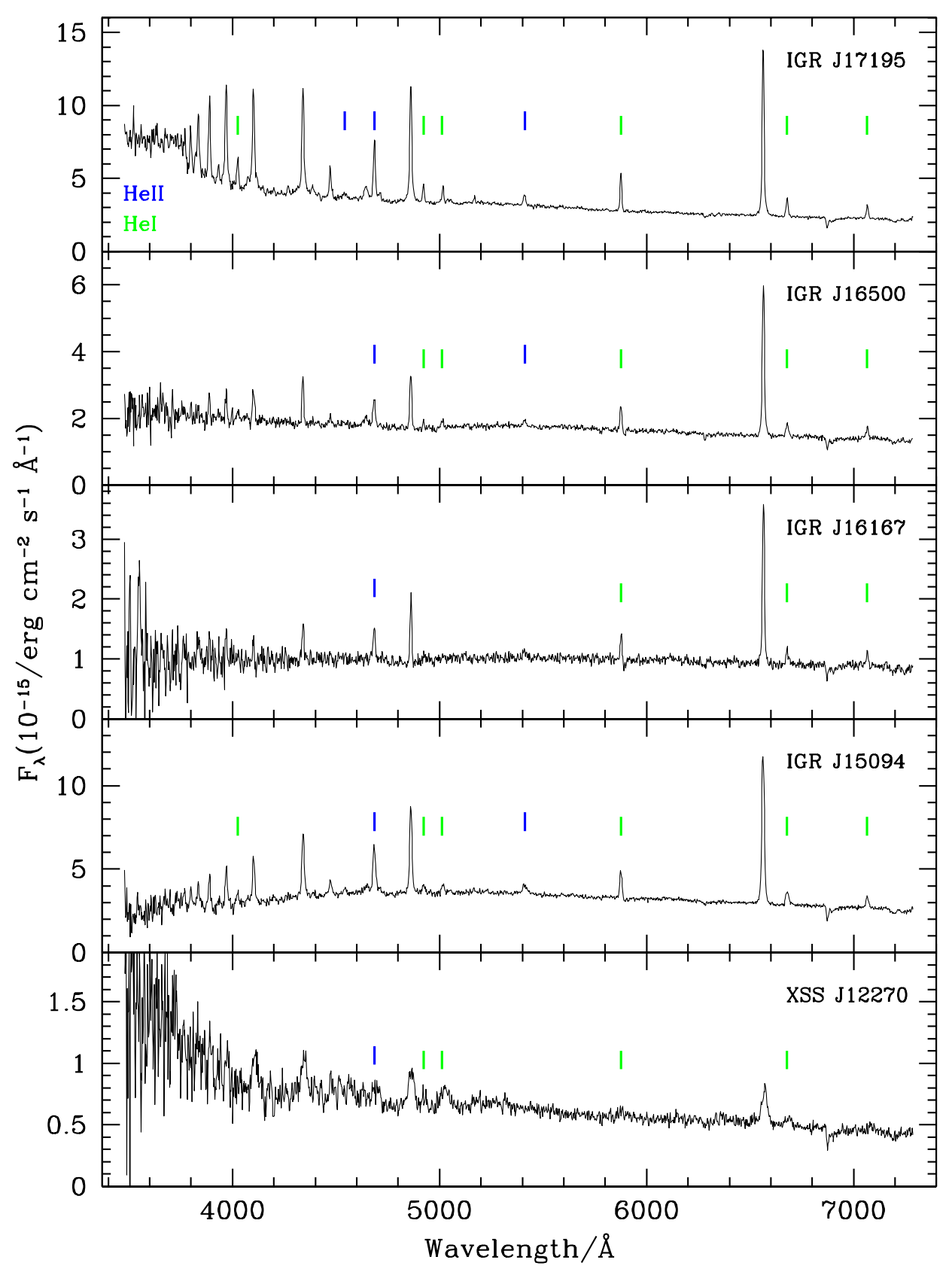

Figure 2: Medium-resolution spectra of the CVs listed in Table 1, obtained with the SAAO 1.9-m telescope. All spectra show Balmer, He I, and He II emission lines. He I and He II lines are marked with green and blue vertical bars, respectively. The $\mathrm{C}$ III/ $\mathrm{N}$ III $\lambda \lambda 4640-4650$ Bowen blend is also detected in the spectra of IGR J15094-6649, IGR J16500-3307, and IGR J17195-4100. The continuum of IGR J15094-6649 is significantly redder than in the discovery spectrum of [30]. 


\subsubsection{IGR J16167-4957}

$13.5 \mathrm{~h}$ of high-speed photometry taken over 3 nights revealed no coherent short-period modulations in the brightness of IGR J16167-4957. The average magnitude found for this system is $V=16.5$; there is no evidence for a systematic change in brightness from the discovery observations of [30].

A spectroscopic orbital period of $5.004 \mathrm{~h}$ was measured for IGR J16167-4957. The orbital signal is not detected in the light curves. There is only one polar known with a period above $5 \mathrm{~h}$ (V1309 Ori); it therefore seems quite unlikely that IGR J16167-4957 will turn out to be a polar.

\subsection{Systems classified as intermediate polars}

The remaining three targets are confirmed as IPs. IGR J15094-6649, IGR J16500-3307, and IGR J17195-4100 all have orbital periods above the period gap, where the majority of IPs are found.

Fourier transforms of the light curves of these three CVs are displayed in the right-hand panels of Fig. 3. The strongest signal is in each case interpreted as the white dwarf spin period; this frequency (and, for IGR J15094-6649 and IGR J17195-4100, the first harmonics) are marked by red vertical bars in Fig. 3. The left-hand panels show all the photometry of these CVs, folded in the spin periods.

\subsubsection{IGR J15094-6649}

I measure $P_{o r b}=5.89 \mathrm{~h}$ and $P_{1}=13.490 \mathrm{~min}$ for IGR J15094-6649. The orbital signal is not detected in the roughly $15 \mathrm{~h}$ of photometry taken of this system. The white dwarf spin modulation is significantly non-sinusoidal.

\subsubsection{IGR J16500-3307}

$P_{\text {orb }}=3.617 \mathrm{~h}$ is found for IGR J16500-3307. The timing of the photometric observations was not ideal-data were taken on 3 separate nights spread over a 7 day baseline, and on another 3 consecutive nights, almost 3 months later. The cycle count between observations is therefore ambiguous. However, the modulation ascribed to the white dwarf spin is detected in all light curves. IGR J16500-3307 has $P_{1}=9.960 \mathrm{~min}$. No harmonics of this modulation are detected, and there is no sign of the orbital frequency in the photometry.

\subsubsection{IGR J17195-4100}

The high-speed photometry of IGR J17195-4100 indicates $P_{1}=18.993 \mathrm{~min}$. In this system the orbital signal, as well as orbital sidebands (see e.g. [41]) and harmonics of the spin and orbital frequencies are detected. The orbital frequency $(\Omega)$ and two of its harmonics, as well as two orbital side bands $(\omega+\Omega$ and $\omega-\Omega)$ are indicated by blue and green vertical bars, respectively, in Fig. 3 .

[33] report candidate periods of $1842 \mathrm{~s}$ and $2645 \mathrm{~s}$ in this source; these are both incommensurate with the white dwarf spin period I find. 

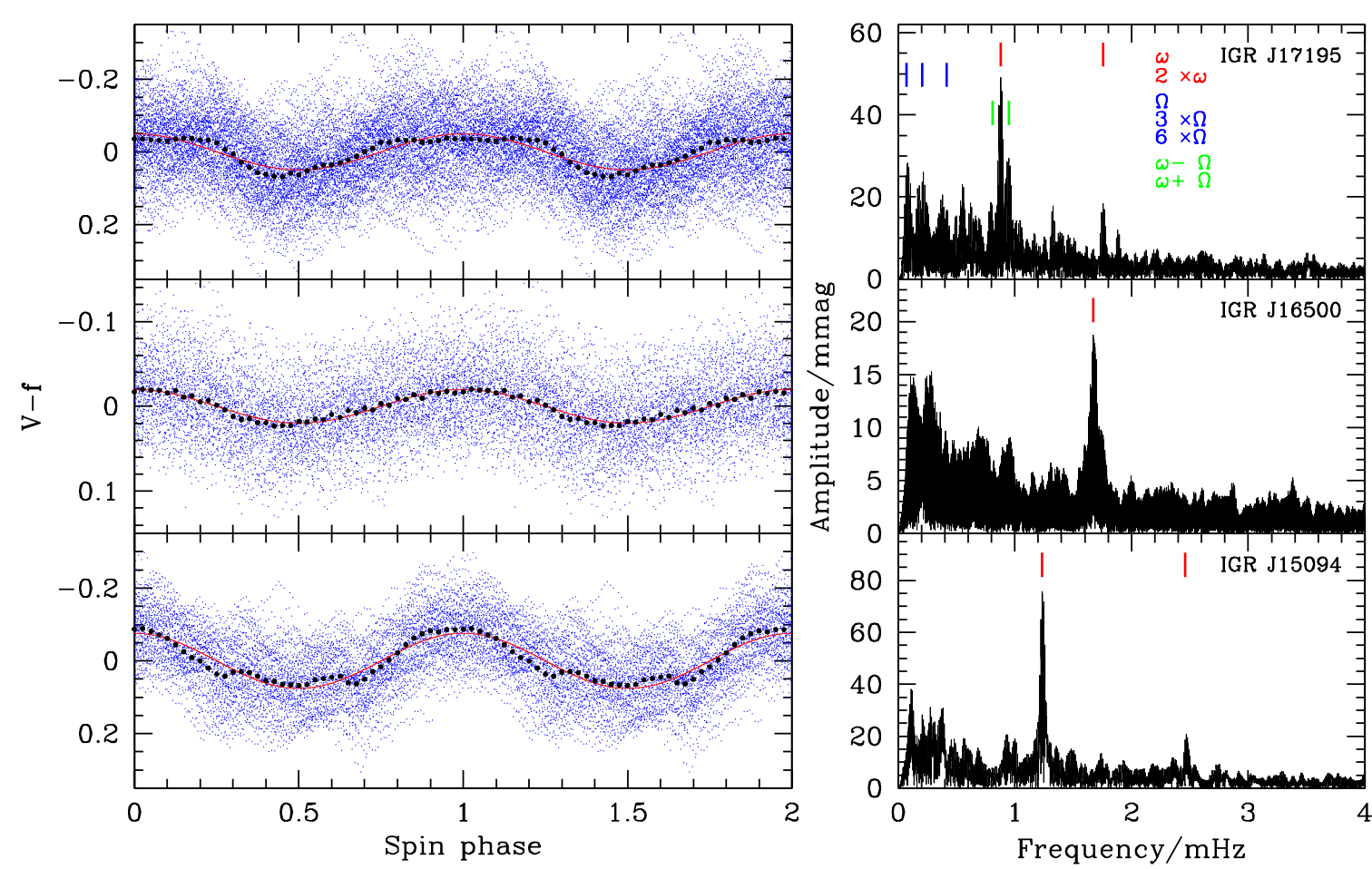

Figure 3: Left: the spin phase folded photometry of IGR J17195-4100, IGR J16500-3307, and IGR J150946649. Blue points are all the data, black points are binned photometry, and the red curves are best-fit sinusoids. Right: Fourier transforms of all the high-speed photometry obtained for each system. The orbital frequency $(\Omega)$ and two harmonics are marked by blue vertical bars, and orbital side bands by green bars, in the Fourier transform of IGR J17195-4100; red bars indicate the white dwarf spin frequency $(\omega)$ in all three Fourier transforms.

\section{Discussion}

INTEGRAL has to date detected $23 \mathrm{CVs}$, of which 13 were not previously known. This is a particularly interesting CV sample -2 of the 6 known asynchronous polars are included, and about 10 systems were known to be IPs, or had been show to be IPs since their detection by INTEGRAL. As a result of this large fraction of IPs found in soft $\gamma$-rays, most CVs identified via INTEGRAL detections are classified as IPs, in the absence of good evidence.

Any study hoping to characterize the $\mathrm{CV}$ population detected in soft $\gamma$-rays will require reliable classifications, as well as orbital period measurements and, in the case of IPs, white dwarf spin period measurements. My high-speed optical photometry confirms only 3 out 5 systems included in this study as IPs.

It has long been known (see e.g. [3]) that the fraction of IPs increases in CV samples selected at shorter wavelengths (as is expected from the hard X-ray spectra and large optical to X-ray flux ratios of IPs). In the [42] sample of all CVs with known orbital periods (a mainly optically selected sample), roughly $7 \%$ are IPs, and $\simeq 12 \%$ of CVs detected at $0.1-2.4 \mathrm{keV}$ (in the ROSAT Bright Survey; [43]) are IPs. This illustrates how rare IPs are in optical and soft X-ray selected samples. At higher energies, however, the fraction increases. $\simeq 40 \%$ of CVs detected in the $1-20 \mathrm{keV}$ band (by the HEAO-1 satellite; [44]) are IPs, and $\gtrsim 50 \%$ of CVs detected at $20-100 \mathrm{keV}$ by INTEGRAL 
are IPs.

Also in terms of the total observed sample of IPs, INTEGRAL is making a significant impactout of about 50 firmly established IPs ([42]), 9 are INTEGRAL discoveries.

\section{Summary}

I have presented optical observations of $5 \mathrm{CVs}$ detected by INTEGRAL. All five have previously been classified as IPs in the literature, but I confirm only 3 (IGR J15094-6649, IGR J165003307, and IGR J17195-4100) of these classifications.

Despite obtaining long light curves of good quality of XSS J12270-4859 and IGR J161674957, no periodic oscillations are detected in these two objects. If the signal found by [33] in XSS J12270-4859 is shown to be coherent, it will mean that this system is an IP; meanwhile, both XSS J12270-4859 and IGR J16167-4957 should be considered a non-magnetic CV.

\section{References}

[1] Warner B., 1995, Cataclysmic Variable Stars. Cambridge Univ. Press, Cambridge

[2] Cropper M., 1990, SSRv, 54, 195

[3] Patterson J., 1994, PASP, 106, 209

[4] Bailey J., Cropper M., 1991, MNRAS, 253, 27

[5] Bailey J., Wickramasinghe D.T., Ferrario L., Hough J.H., Cropper M., 1993, MNRAS, 261, L31

[6] Cropper M., et al., 1989, MNRAS, 236, 29P

[7] Bailey J., Ferrario L., Wickramasinghe D.T., 1991, MNRAS, 251, 37P

[8] Wickramasinghe D.T., Cropper M., Mason K.O., Garlick M., 1991, MNRAS, 250, 692

[9] Lamb F.K., Aly J.-J., Cook M.C., Lamb D.Q., 1983, ApJ, 274, L71

[10] Campbell C.G., 1989, MNRAS, 236, 475

[11] Stockman H.S., Schmidt G.D., Lamb D.Q., 1988, ApJ, 332, 282

[12] Campbell C.G., Schwope A.D., 1999, A\&A, 343, 132

[13] Schmidt G.D., Stockman H.S., 1991, ApJ, 371, 749

[14] Bath G.T., Evans W.D., Pringle J.E., 1974, MNRAS, 166, 113

[15] Lamb D.Q., 1974, ApJ, 192, L129

[16] Patterson J., Robinson E.L., Nather R.E., 1978, ApJ, 224, 570

[17] Ghosh P., Lamb F.K., 1979, ApJ, 234, 296

[18] Penning W.R., Schmidt G.D., Liebert J.E., 1986, ApJ, 301, 885

[19] Stockman H.S., Berriman G., Liebert J., Moore R.L., Whickramasinghe D.T., 1992, ApJ, 401, 628

[20] Buckley D.A.H., Sekiguchi K., Motch C., O’Donoghue D., Chen A.-L., Schwarzenberg-Czerney A., Pietsch W., Harrop-Allin M.K., 1995, MNRAS, 275, 1028

[21] Rodríguez-Gil P., Potter S.B., 2003, MNRAS, 342, L1 
[22] Bird A. J., et al., 2006, ApJ, 636, 765

[23] Bird A. J., et al., 2007, ApJS, 170, 175

[24] Barlow E.J., Knigge C., Bird A.J., J Dean A., Clark D.J., Hill A.B., Molina M., Sguera V., 2006, MNRAS, 372, 224

[25] Revnivtsev M., Sazonov S., Krivonos R., Ritter H., Sunyaev R., 2008, A\&A, 489, 1121

[26] Araujo-Betancor S., Gänsicke B. T., Hagen H.-J., Rodriguez-Gil P., Engels D., 2003, A\&A, 406, 213

[27] Staude A., Schwope A. D., Krumpe M., Hambaryan V., Schwarz R., 2003, A\&A, 406, 253

[28] Gänsicke B. T., et al., 2005, MNRAS, 361, 141

[29] Bonnet-Bidaud J. M., Mouchet M., de Martino D., Silvotti R., Motch C., 2006, A\&A, 445, 1037

[30] Masetti N., et al., 2006, A\&A, 459, 21

[31] Masetti N., et al., 2008, A\&A, 482, 113

[32] Landi R., Bassani L., Dean A. J., Bird A. J., Fiocchi M., Bazzano A., Nousek J. A., Osborne J. P., 2008, in press (arXiv:0810.1844)!

[33] Butters O. W., Norton A. J., Hakala P., Mukai K., Barlow E. J., 2008, A\&A, 487, 271

[34] O’Donoghue D., 1995, BaltA, 4, 519

[35] Stone R.P.S., Baldwin J.A., 1983, MNRAS, 204, 347

[36] Horne K., 1986, PASP, 98, 609

[37] Patterson J., 1981, ApJS, 45, 517

[38] Warner B., Woudt P. A., Pretorius M. L., 2003, MNRAS, 344, 1193

[39] Warner B., 2004, PASP, 116, 115

[40] Pretorius M. L., Warner B., Woudt P. A., 2006, MNRAS, 368, 361

[41] Warner B., 1986, MNRAS, 219, 347

[42] Ritter H., Kolb U., 2003, A\&A, 404, 301 (update RKcat7.10)

[43] Schwope A.D., Brunner H., Buckley D., Greiner J., Heyden K.v.d., Neizvestny S., Potter S., Schwarz R., 2002, A\&A, 396, 895

[44] Silber A. D., 1992, PhD Thesis, Massachusetts Institute of Technology 